\title{
Structural spacing and the determination of habitat complexity: examining the Bartholomew et al. (2000) index
}

\author{
Susan S. Bell*, Robert A. Brooks, William E. Ellis \\ Department of Biology, University of South Florida, Tampa, Florida 33620-5200, USA
}

\begin{abstract}
We evaluate the use of the Bartholomew Sp/Pr index with reference to the collection of information to be used in index calculation and interpretation. Through examination of hypothetical structural arrangements, we address issues related to determining the spacing between structures which are non-uniformly distributed. We also utilize fieldcollected data to examine how measures of inter-structural spacing may vary when (1) 2 different field methods are used and (2) measurements of the same structures are made over different spatial scales. Using a mangrove prop-root system, measures of inter-structural spacing within $2900 \mathrm{~m}^{2}$ areas using a 100 point confined arc method were obtained, as were small quadrat samples conducted within the same sites using the confined arc and nearest neighbor method. In small quadrat sampling, mean between-root distances using the arc method were nearly $20 \%$ less than those obtained from the $900 \mathrm{~m}^{2}$ plots. The greatest difference in spacing measurements, and accordingly $\mathrm{Sp}$ values, was recorded when the confined arc and nearest neighbor methods were applied to the same inter-structural spaces. Our combined results illustrate that quantification of spatial arrangement can vary with both method and scale of measurement and suggest that across-study comparisons using the index developed by Bartholomew et al. (2000, Mar Ecol Prog Ser 206:45-58) will be difficult to interpret unless the same method of structural complexity measurement is employed, and similar spatial arrangements are compared.
\end{abstract}

KEY WORDS: Habitat complexity - Inter-structural space · Mangrove $\cdot$ Nearest neighbor $\cdot$ Prop root $\cdot$ Scale

Resale or republication not permitted without written consent of the publisher

In a recent paper, Bartholomew et al. (2000) proposed a new index of structural habitat complexity for use in studies that assess predator foraging in highly structured habitats. The index, calculated as the ratio of distance between structural elements within a habitat $(\mathrm{Sp})$ to dimensions of predator width (Pr), provides some measure of foraging success of a predator under different structural complexities. The authors argued that any inter-structural spacing that was smaller than a predator's width should result in interference with

*Email: sbell@chuma1.cas.usf.edu predator/prey interactions. The authors also argued that the index, which is unitless, would invite comparison of results across studies and application to any habitat scale or type.

Overall, the applicability of the Bartholomew et al. (2000) index was best supported from their laboratory studies within which structural complexity could be easily controlled. Bartholomew et al. (2000) tested the $\mathrm{Sp} / \mathrm{Pr}$ index using a fish predator (Fundulus heteroclitus) and benthic prey typically found among southeastern, USA, salt marsh Spartina alterniflora vegetation. In a series of laboratory studies on fish predation, with uniformly spaced wooden dowels as simulated structure in small aquaria, they consistently found that benthic prey survivorship was increased in treatments of highest dowel density and smallest inter-dowel space, supporting the applicability of their index. Curiously, their results from a limited field study did not support the concept that differences in inter-structural spacing among Spartina alterniflora resulted in changes in the sizes of the target fish predator utilizing the marsh.

The disparity in the results found between the laboratory and field setting may be attributed to the differences in the spatial distribution of the structural components. Unlike the laboratory set-up of Bartholomew et al. (2000), natural structures are rarely uniformly spaced and may display a variety of spatial arrangements. Indeed, an index based upon a 'mean' assessment of spacing may vary in its efficacy to describe the structural complexity of a setting, particularly if a great deal of variation among the magnitude of spaces between objects exists. For example, in Fig. 1 we present theoretical spatial arrangements of objects with 3 different dispersions - uniform, random and clumped. In all cases, the mean value for the inter-structural spaces are equivalent according to the method described by Bartholomew et al. (2000). Clearly, however, summary statistics fail to capture some potentially important differences in spacing among structures, specifically in 

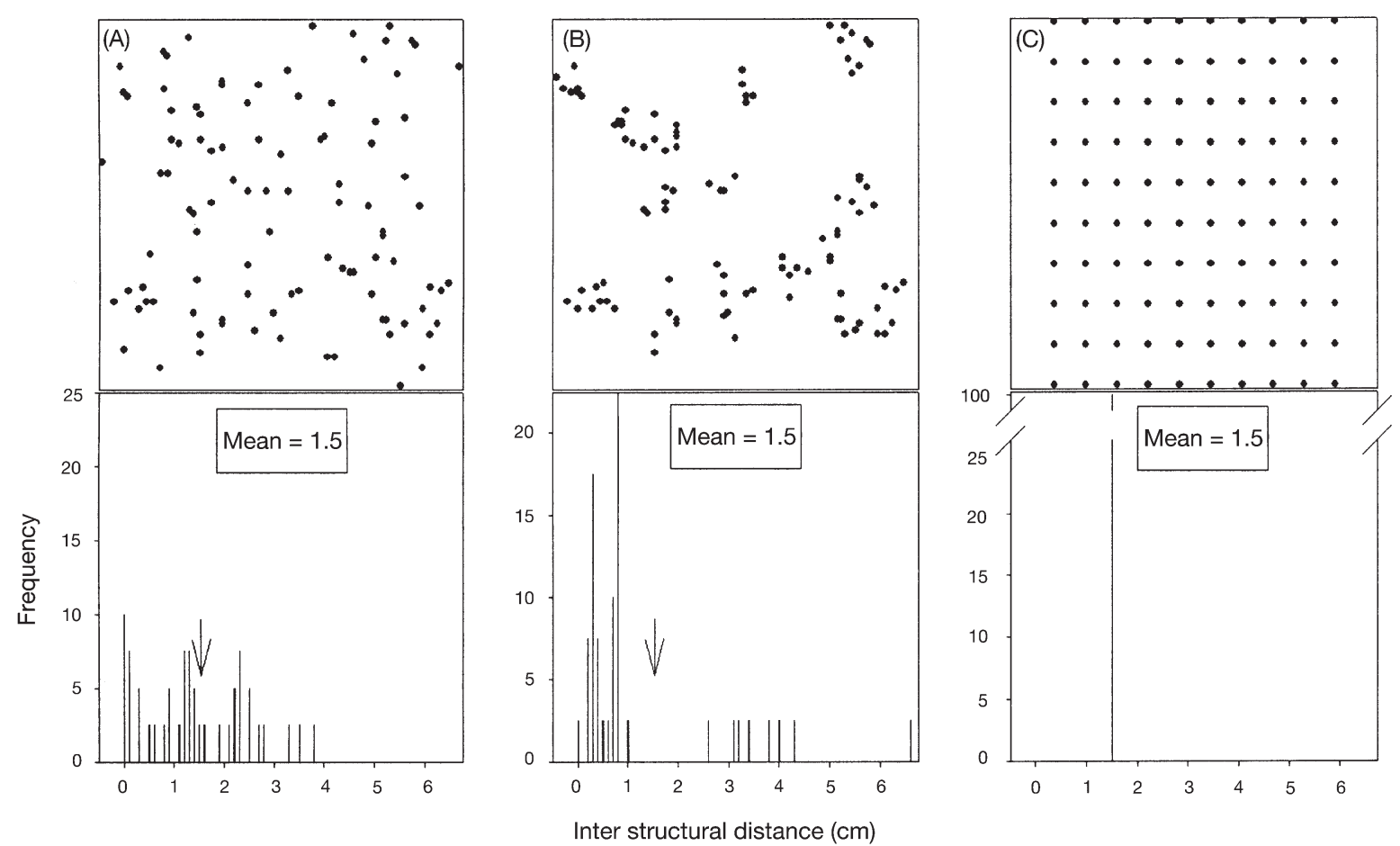

Fig. 1. Hypothetical structural arrangements that display (A) random, (B) clumped, and (C) uniform arrangement of 100 objects, and the corresponding frequency distribution of spacing units for each arrangement. The Bartholomew et al. (2000) arc method using 40 randomly chosen points was used to generate the inter-structural distributions. Note that the mean spacing unit (= interstructural space) is identical for each structural arrangement. Arrows in (A) and (B): mean interstructural space

the non-uniform distributions (see Fig. 1). Comparison of the distribution of spacing among the 3 spatial arrangements in Fig. 1 indicates that 57.5 versus $75.0 \%$ of spaces in the random and clumped distributions, respectively, are less than the mean value. Such differences in the distribution of spaces, lost by calculation of an index, may be of critical interest when comparing predator activity in structured habitats across studies.

Materials and methods. Application of this index in non-uniformly structured habitats may be further complicated by the choice of the method used for estimating dimensions of interstices. We conducted a study using a variety of methods to quantify spacing of root structures in a subtropical mangrove-rimmed estuary in Rookery Bay, on the southwest Florida coast (260 $\left.01^{\prime} 51^{\prime \prime} \mathrm{N}, 81^{\circ} 44^{\prime} 51^{\prime \prime} \mathrm{W}\right)$. Rhizophora mangle L., the red mangrove, dominates this area and has a distinctive aboveground system of prop and drop roots. These aboveground roots (5 to $10 \mathrm{~mm}$ diameter), once attached to the substrate, undergo multiple lateral branching producing a haystack like appearance (Gill \& Tomlinson 1975) and spacing between roots can be easily measured. Unlike the uniform structural arrangement utilized by Bartholomew et al. (2000), mangrove roots at the $1 \mathrm{~m}^{2}$ scale generally display random or clumped distributions (Brooks 2001).
We employed the method of Bartholomew et al. (2000) to our field site to determine the distribution of between-root spaces within $2 \times 900 \mathrm{~m}^{2}$ areas of fringing mangroves in July 2001. Two plots were demarcated such that they extended from the shoreline to $30 \mathrm{~m}$ inland through the mangroves, as this intertidal region represented the tidal extent (and thus probably limit of predator and prey access) within the area. Following the field procedure of Bartholomew et al. (2000), 100 random points were selected within the plot and the closest root to each point was tagged. The distance $(\mathrm{cm})$ from this tagged root to the next closest root confined within a randomly chosen $90^{\circ}$ arc (= confined arc-large plot method) was then recorded.

We then utilized 2 different methods for determining 'between structure' spaces and, subsequently, the $\mathrm{Sp} / \mathrm{Pr}$ index. Small quadrat sampling was performed in July 2001 within the same sites in which the previous 100 point surveys were conducted. Ten quadrats were randomly placed within each $900 \mathrm{~m}^{2}$ area. Size of quadrats $\left(68 \mathrm{~cm}^{2}\right.$ for Site $1 ; 73 \mathrm{~cm}^{2}$ for Site 2$)$ was determined such that each quadrat was large enough to include $95 \%$ of the between-root distances found from the 100 point sample survey discussed above. For every root within a quadrat, 2 different measures were taken. The first method used was the commonly employed dis- 
tance between the selected root and its nearest neighboring root (= nearest neighbor method; Krebs 1999). The second measured distance was to the nearest neighbor confined within a randomly chosen $90^{\circ}$ arc (= confined arc-small quadrat method); this allowed for the nearest root to reside outside of the quadrat.

Results and discussion. The mean values for interroot spacing using different methods varied, even within the same sampling site (Table 1). At both sites, the confined arc method using 100 points had the largest value of mean spacing, while the nearest neighbor values were consistently smallest. Importantly, the utilization of mean spacing measures recorded from the confined arc-large plot and small quadrat methods at Sites 1 and 2 produced $\mathrm{Sp} / \mathrm{Pr}$ index values that were 2.6 to $2.8 \times$ larger than those calculated using the nearest neighbor method, greatly influencing what might be considered predator accessibility for foraging within the mangrove root system.

The arc and nearest neighbor methods produced different values for the inter-structural spaces from the same area in the small quadrat study (Table 1). These discrepancies in results emerged because restrictions upon which structures were considered to be 'neighbors' influenced the mean spacing value that was obtained. When we calculated the inter-structural spacing using the confined arc and nearest neighbor methods for 40 randomly chosen points in the theoretical patterns presented in Fig. 1, we found identical values $(1.5 \mathrm{~cm})$ for both methods for the uniform spatial pattern, but widely different values in the clumped $(0.29 \mathrm{~cm}$; nearest neighbor; $1.5 \mathrm{~cm}$ confined arc) and random $(0.67 \mathrm{~cm}$ nearest neighbor; $1.5 \mathrm{~cm}$ confined arc) distributions. These results combine to illustrate that across-study comparisons using the index developed by Bartholomew et al. (2000) will be difficult to interpret unless the same method of structural complexity measurement is employed or uniform distributions are the norm.

While we address issues linked to spatial arrangement in this discussion, we also acknowledge that interpreting patterns of complexity is intimately linked to spatial scale (McCoy \& Bell 1990). Problems with determining the appropriate spatial scale over which one should collect information for characterization of structural complexity remain formidable. We found that in small quadrat sampling of highly clumped mangrove roots, mean between-root distances using the confined arc method were nearly $20 \%$ less than those obtained from the same site using the 100 point method. Whether such discrepancies are trivial or critically important can only be determined by the question of interest and the predator sizes to be included in the index calculation. Information on features such as area over which predators move and/or prey utilization

Editorial responsibility: Kenneth Heck (Contributing Editor), Dauphin Island, Alabama, USA
Table 1. Rhizophora mangle. Mean distance (cm) between roots at study sites using the arc versus nearest neighbor method of measurement size of sampling scale

\begin{tabular}{|lrr|}
\hline Method & Site 1 & Site 2 \\
\hline Arc (large plot) & 19.5 & 23.4 \\
Arc (small quadrats) & 15.9 & 18.9 \\
Nearest neighbor (small quadrats) & 5.6 & 5.9 \\
\hline
\end{tabular}

of, or distribution around, structure, if available, could help delineate an appropriate spatial scale of study for calculating the Sp value for the Bartholomew et al. (2000) index.

In conclusion, calculation of the Bartholomew et al. (2000) index provides an intuitively attractive approach to evaluate the potential impact of habitat complexity on predator foraging. While the measurement of predator dimension to be utilized in the index is straightforward, determining the most appropriate method of measurement of structural spacing may be problematic, especially when spatial arrangements of structures are non-uniform. Bartholomew et al. (2000) extol their index as appealing because it is dimensionless, can be applied across all habitat types and may allow easy comparison of results across habitats. Our findings suggest that an easy comparison may not be the case, as the 'across habitat' use of the index may be limited to instances where similar methods are used to quantify spacing, spatial arrangements are similar, and the issue of scale is addressed.

Acknowledgements. This study was supported in part from an Aylesworth Fellowship to R.A.B. and a NOAA-NERRS Graduate Fellowship to W.E.E.

\section{LITERATURE CITED}

Bartholomew A, Diaz RJ, Cicchetti G (2000) New dimensionless indices of structural habitat complexity: predicted and actual effects on a predator's foraging success. Mar Ecol Prog Ser 206:45-58

Brooks RA (2001) Plant-animal interaction within the red mangroves, Rhizophora mangle L., of Tampa Bay; mangrove habitat classification and isopod, Sphaeroma terebrans Bate, colonization of a dynamic root substratum. $\mathrm{PhD}$ thesis, University of South Florida, Tampa

Gill AM, Tomlinson PB (1975) Aerial roots: an array of forms and functions. In: Torrey JG, Clarkson DT (eds) The development and function of roots. Academic Press, New York, p 237-260

Krebs CJ (1999) Ecological methodology. Benjamin Cummings, Menlo Park, CA

McCoy ED, Bell SS (1990) Habitat structure: an overview. In: Bell SS, McCoy ED, Mushinsky HR (eds) Habitat complexity: the physical arrangement of objects in space. Chapman \& Hall, New York

Submitted: November 19, 2002; Accepted: December 5, 2002 Proofs received from author(s): January 30, 2003 